\title{
Neglected posterior acetabular wall fracture managed with uncemented total hip arthroplasty with posterior wall reconstruction by femoral head autograft: a case report
}

\author{
Ram Avtar, Varun Khanna*, Krishan Kumar
}

Department of Orthopaedics, ESI Hospital, PGIMSR, Basaidarapur, Ring Road, New Delhi, India

\author{
Received: 05 March 2018 \\ Revised: 29 March 2018 \\ Accepted: 30 March 2018 \\ *Correspondence: \\ Dr. Varun Khanna, \\ E-mail:varun0609@yahoo.com
}

Copyright: () the author(s), publisher and licensee Medip Academy. This is an open-access article distributed under the terms of the Creative Commons Attribution Non-Commercial License, which permits unrestricted non-commercial use, distribution, and reproduction in any medium, provided the original work is properly cited.

\begin{abstract}
Total hip arthroplasty (THA) poses several challenges as a treatment option for acetabular fracture especially in the setting of a neglected fracture. The potential technical challenges of THA include the altered anatomy and hip centre, acetabular bone deficit, difficulty in achieving long-term fixation of the acetabular shell and risk of nerve injury. In our case, a forty two years old, male labrourer presented with a five months old injury, which was previously managed only conservatively. Preoperative evaluation revealed displaced posterior wall acetabulum with superiorly migrated femur head with flattened contour. We managed the case with uncemented THA. The acetabular defect was addressed using the femoral head autograft which was secured with screws. On three months follow up, radiographical evalution showed good graft consolidation without any acetabular component migration and functionally a Harris hip score of 76. Thus, in case of neglected acetabular fractures, with high probability of post traumatic arthritis to develop, THA remains the preferred choice of management. Albeit challenging to perform, THA provides effective way to decrease pain, improve stability and increase functional outcomes.
\end{abstract}

Keywords: Acetabular fracture, Total hip arthroplasty, Neglected, Posterior wall

\section{INTRODUCTION}

Although there has been many recent advances in the management of the acetabular and pelvic fractures over decades, but still the delay in its management due to various reasons, is present in both developing and nondeveloping nations. ${ }^{1}$ The delay of surgical intervention has shown to have a detrimental effect on the overall outcome in both the elementary and associated fracture patterns. $^{2}$

Total hip arthroplasty (THA), after acetabular fracture is warranted in two distinct settings; firstly after an acute acetabular fracture which predictablely has poor outcome with ORIF and secondly (and more commonly) after the patient develops post traumatic arthritis with either previous operative or conservative management. Post traumatic arthritis of the hip can develop in $12-57 \%$ of patients after an acetabular fracture. Once it develops, salvage treatment options include arthroplasty or arthrodesis. ${ }^{3}$ Whereas any impaction injury of the femur head or acetabulum or any full thickness abrasive loss of articular cartilage after injury constitutes an absolute indication for THA. ${ }^{4}$

While treating such fractures with THA, problems associated with neglected acetabular fractures such as cavitary bone defect or peripheral bone defect fracture non-union, hip dislocation, protrusio, have to be considered. Our case report is of a neglected (five months 
old), displaced posterior wall fracture acetabulum with dislocated hip. The case was managed by cementless total hip arthroplasty with posterior wall reconstruction using femoral head autograft.

\section{CASE PRESENTATION}

A forty two year old labrourer presented with pain and tenderness in the left hip joint after sustaining a fall while unloading goods from a truck, five months back. Patient was unable to bear weight there on. He had taken treatment at a local hospital in the form of only symptomatic management and longitudinal traction. On examination, the limb was fixed in internal rotation deformity with restricted movement in the two planes. There was shortening of $3 \mathrm{cms}$ on the affected side with high riding trochanter. There was associated nerve palsy presenting as foot drop. The skin traction on which the patient had come was replaced by skeletal traction with increased weight. Preoperative evaluation included x-rays in AP view (Figure 1) and computed tomography both 2D (Figure 2) and 3D reconstruction (Figure 3).

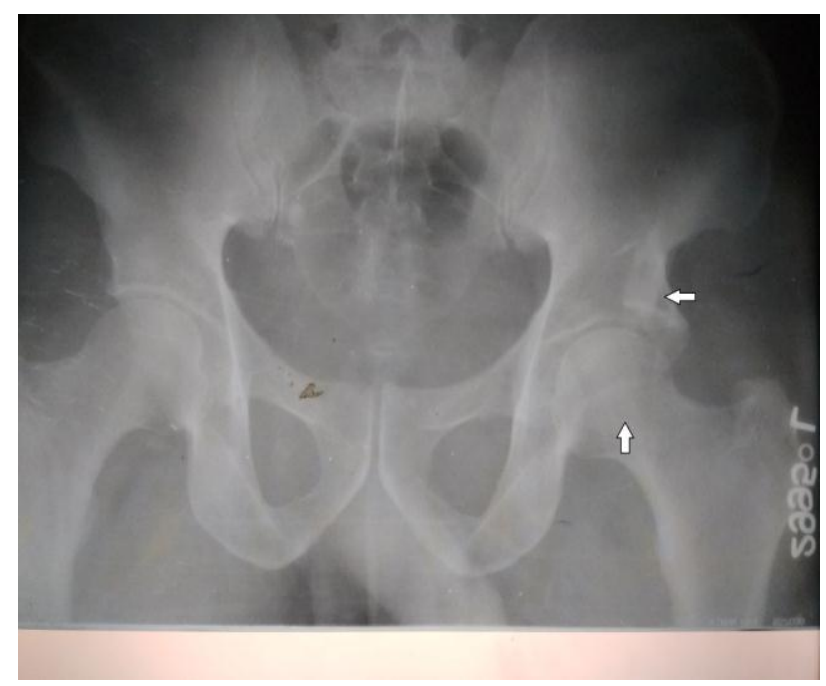

Figure 1: X-ray pelvis with $\mathrm{B} / \mathrm{L}$ hip showing deficient and displaced posterior wall (arrows).

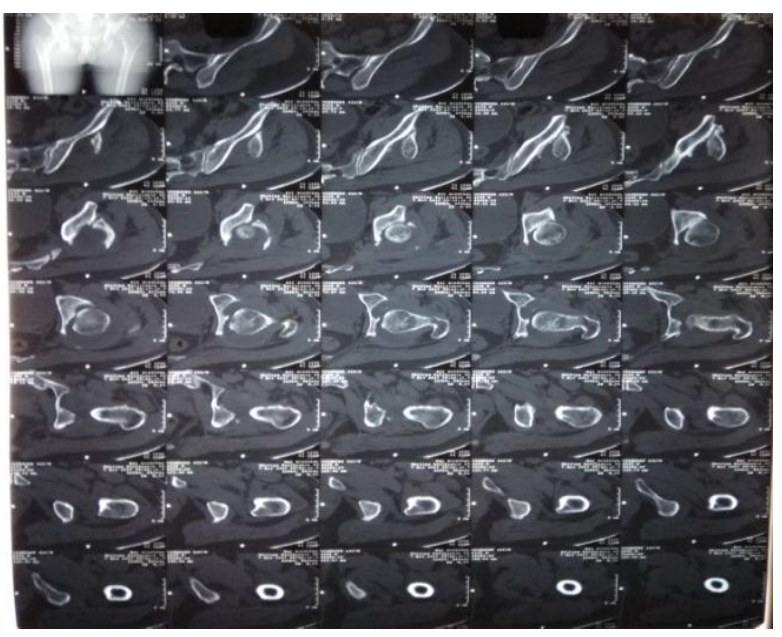

Figure 2: CT scan 2D reconstruction.

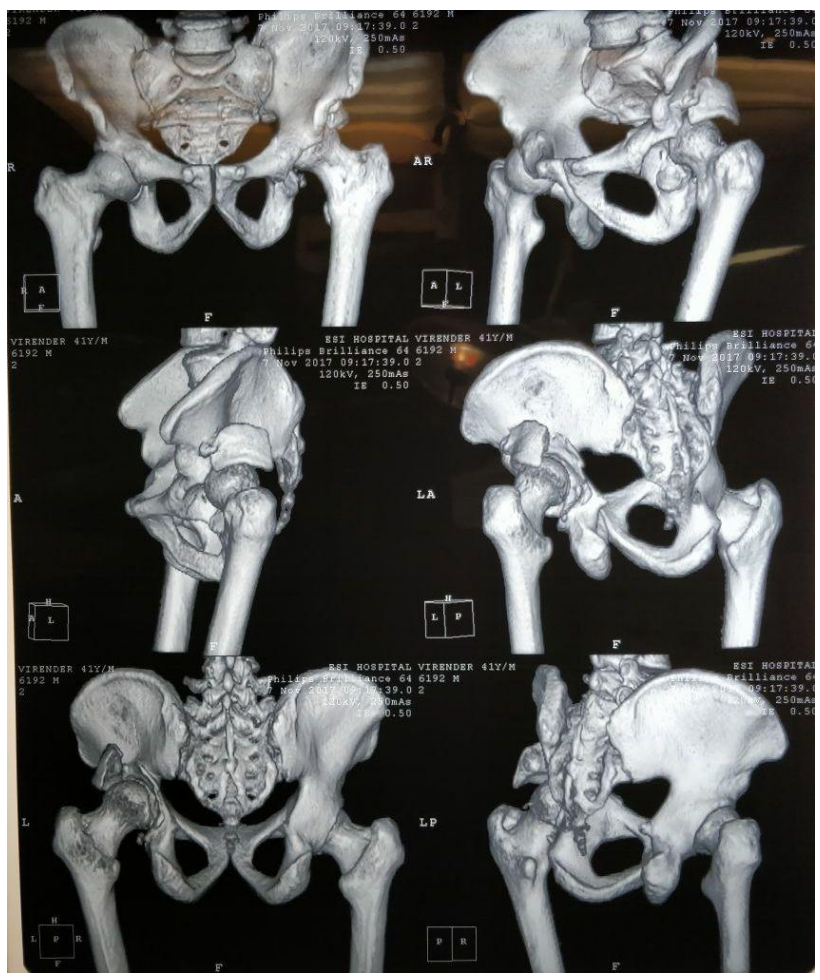

Figure 3: CT scan 3D reconstruction.

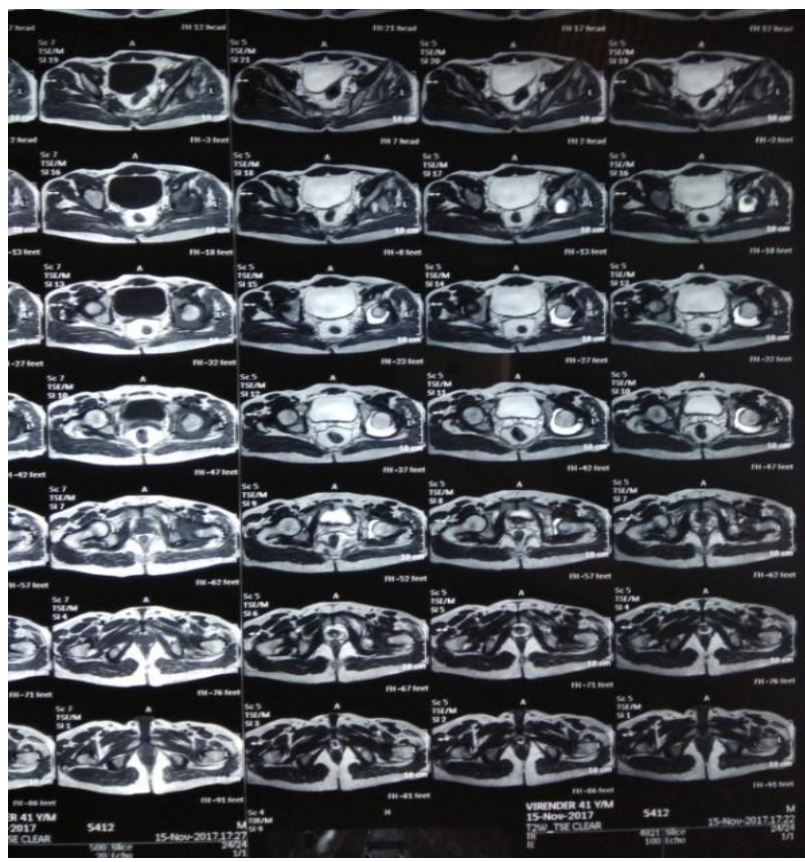

Figure 4: MRI hip joint (axial cut) showing marrow oedema and joint effusion.

3D computed tomography scan provided a clear view about the acetabular and periacetabular bony anatomy. To rule out any arthritic changes in the femoal head, MRI of the hip joint (Figure 4 and 5) was done which revealed flattening of the head, joint effusion bone marrow oedema, confirming the post traumatic injury of the head. 


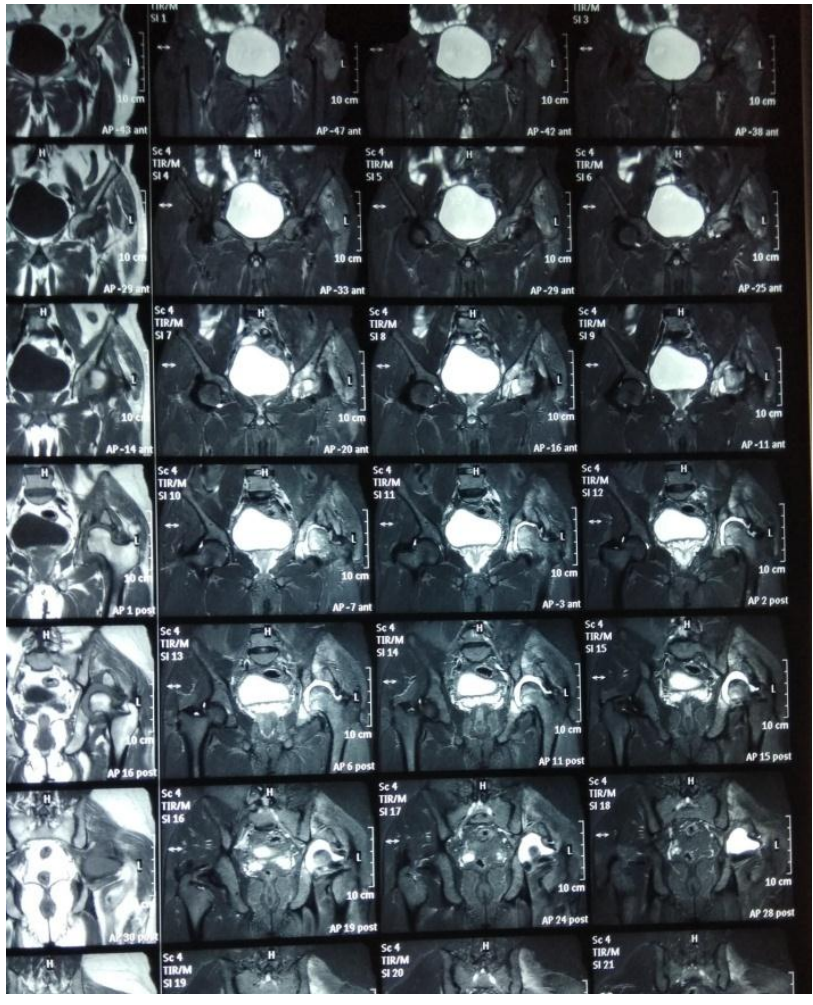

Figure 5: MRI hip (coronal cut) showing flattening of the head, joint effusion, bone marrow oedema.

\section{Surgical technique}

Patient was placed in the lateral decubitus on the radiolucent table, posterolateral approach was used. After careful dissection into the deeper layers, the head was dislocated. Femoral osteotomy was done in standard fashion and femoral head was saved for later as use of autograft. The head revealed large impaction injury with focal cartilage loss (Figure 6). The acetabular margins were clearly exposed to reveal posterosuperior defect of the wall. The defect was restored by creating a contoured wall support with the help of femoral autograft (Figure 7). The head was denuded from its cartilage and then fixed adjacent to the deficient posterior edge of the remaining acetabular cavity. The graft was secured onto the acetabulum with help of long $4.5 \mathrm{~mm}$ cancellous screws.

The graft was then gently reamed with the acetabular reamer and the constructed cavity became one unit and was sequentially reamed thereon (Figure 7). The direction of the screw were directed such that they do not interfere with the reaming. After this the porous coated acetabular shell (Pinnacle Hip Solution by Depuy Synthes) of size $46 \mathrm{~mm}$ was fixed with cancellous bone screws in the reconstructed cavity. The shell placement was checked with intraoperative fluoroscopy (Figure 8)

Polyethylene liner of size $28 \mathrm{~mm}$ was fitted. Femoral canal was then prepared and cementless femoral stem (HA coated) of size 11 was placed. Ceramic head of size
$28 \mathrm{~mm}$ was used and hip was reduced. The hip was stable with a good range of movement. The rotators were reattached to the posterior greater trochanter through drill holes (Figure 9).

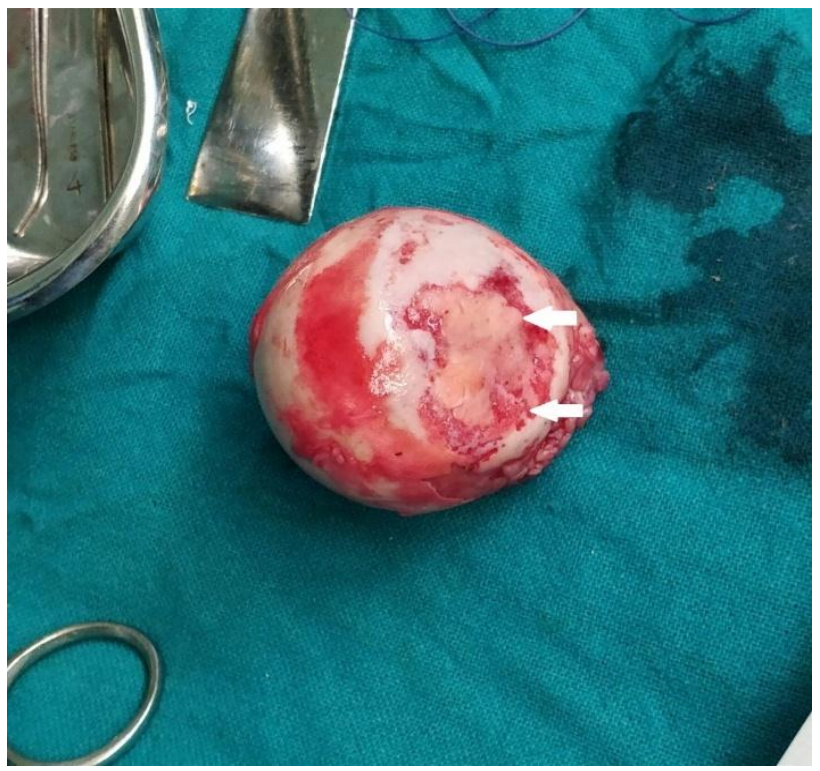

Figure 6: Post traumatic impaction injury showing destruction of the articular surface (arrow).

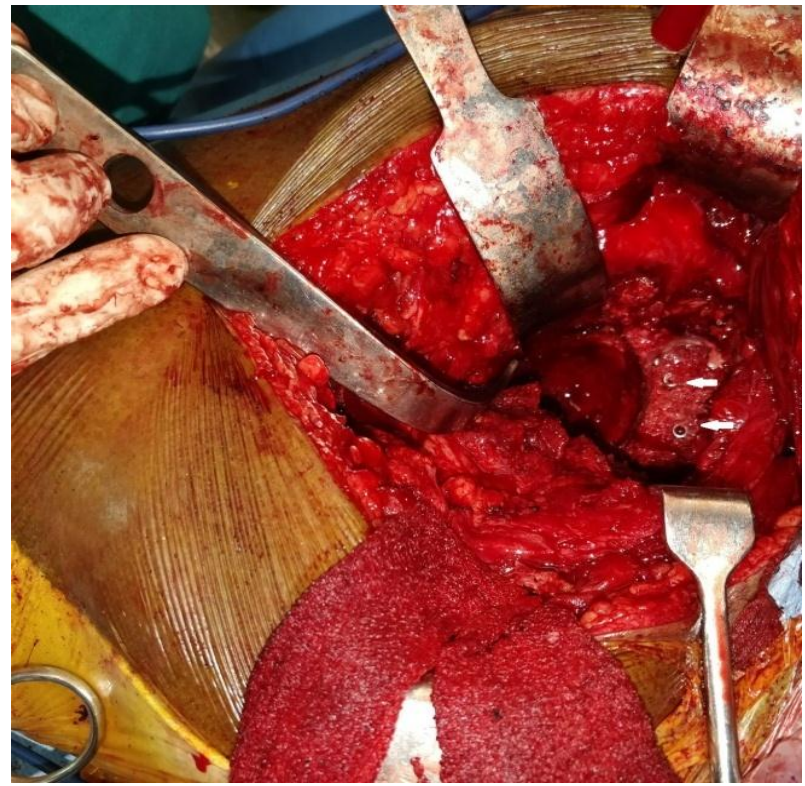

Figure 7: Posterior wall reconstruction after contouring of the femoral head and fixation into the ilium with screws (arrow).

Range of movement exercises started postoperatively, with non-weight bearing for one month. Toe-touch weight bearing started after one month. By the end of three months, the patient was allowed complete weight bearing. Foot drop was managed by physiotherapy and orthotics. At three months follow up (Figure 10), autograft showed good uptake and patient had good functional outcome with Harris hip score of 76. 


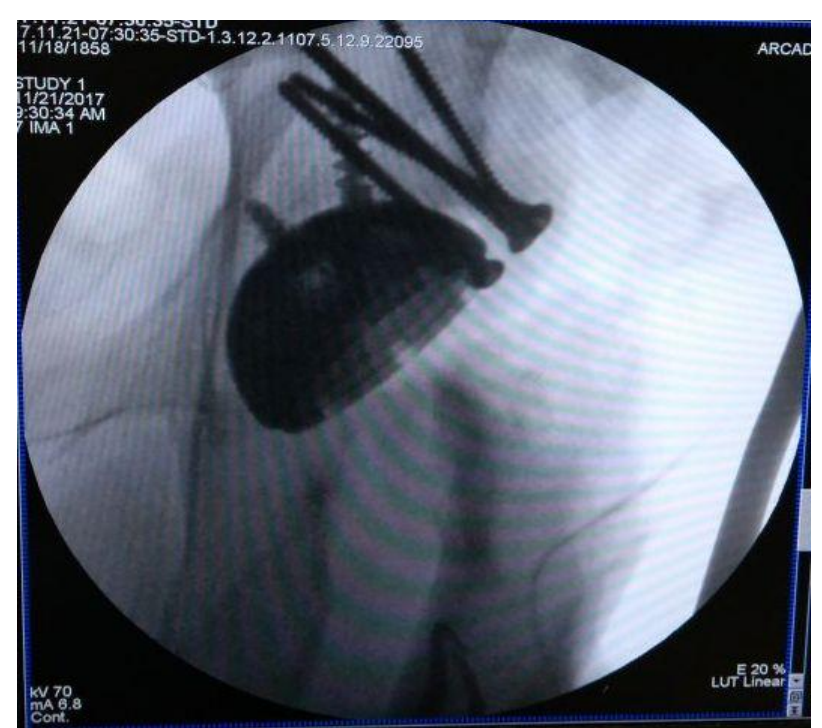

Figure 8: Intraoperative fluoroscopy.

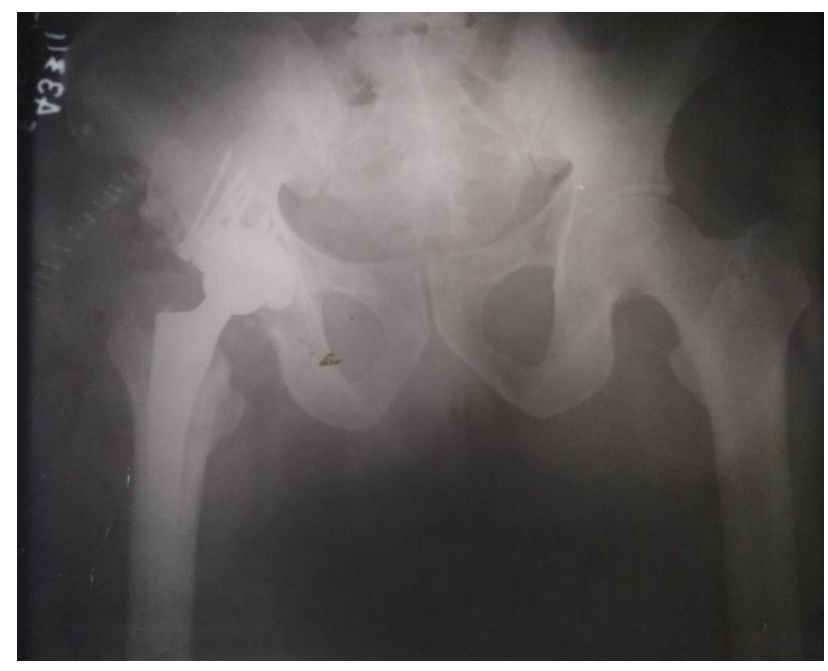

Figure 9: Immediate postoperative $\mathrm{X}$-rays.

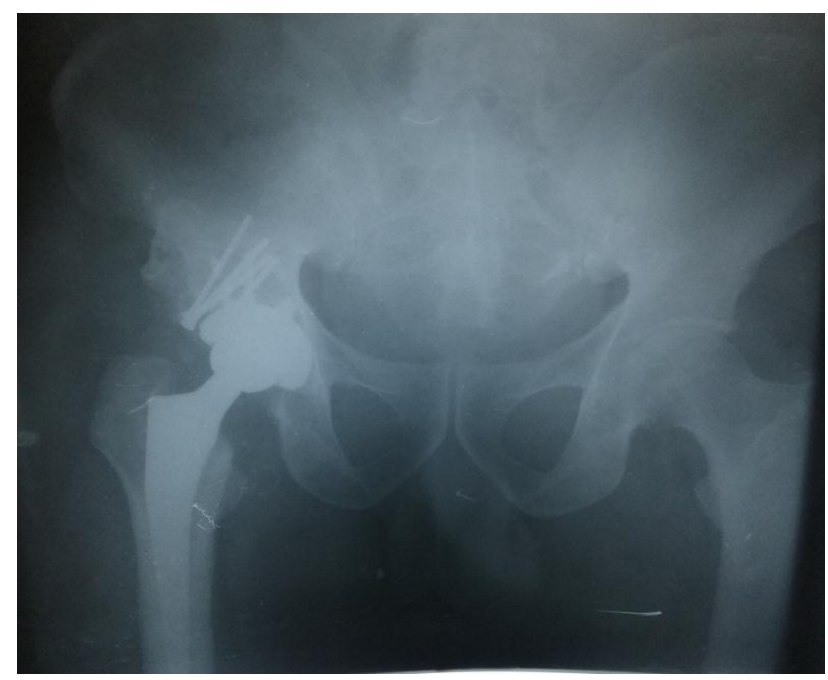

Figure 10: 3 months follow up X-rays, showing good autograft integration.

\section{DISCUSSION}

The term 'neglected acetabulum' has not been well defined in the literature but while evaluating the consequence of delay in surgery, Letournel reported that acetabular fracture, which remains untreated for more than 3 weeks has poor clinical and radiological outcome. ${ }^{5}$ The delay in the management has been attributed to varied reasons. In a study, Bircher et al found reasons such as lack of capacity of tertiary centres in the form of fully trained pelvic surgeons or incapacity of the centre in the lack of Intensive care unit beds. ${ }^{1}$ The discrepency in the referral system due lack of strict transfer criterion causes these patients to be eventually treated by non pelvic specialists and end up getting mismanaged.

In a country like India, the reason for delay may vary from economic constraints to poor medical health, osteoporosis to poor soft tissue conditions or open injuries or communited fracture. ${ }^{6}$ So, the awareness and sense of urgency of early treatment of these injuries is still lacking and must be raised as not only it affects the long term outcome but also makes the eventual surgery a difficult task.

At later stages, ORIF in such cases may not be an ideal solution because of the altered anatomy, impaction injury to femoral head damage due to pressure by the fractured acetabular edge, avascular necrosis, callus formation causing difficult mobilization of the displaced fragment, difficulty in indirect reduction of the fracture fragments and macerated acetabular fragments all contributing to inadequate fracture reduction. Majority of such fractures are now treated with total hip replacement. ${ }^{7}$

Hamlin et al, in their study describe factors predicting development of post traumatic osteoarthritis in acetabular fractures which include cartilaginous damage, hip instability, age $>40 y r s$, posterior acetabular wall involvement and initial displacement $>20 \mathrm{~mm}^{7}$ In the case of impaction injury of the head of femur and full thickness abrasive loss of the articular cartilage, THA is an absolute indication.

Overall, literature shows that both cemented and uncemented arthroplasty has been tried in case of neglected acetabular fractures with varied success. Studies for uncemented fixation by Berry et al, has not shown promising results with 9 out of 34 acetabuli implanted going for revision after a minimum of 10 years follow-up, while other studies for uncemented THA have good to excellent results. ${ }^{8}$ Bellabarba et al, studied 30 hips replaced for post traumatic arthritis due to acetabular fracture and found 10-year survival (revision or radiographic loosening as the end point) of $97 \%$, similar to the results of primary total hip replacement for nontraumatic arthritis. ${ }^{9}$

But most studies emphasized that the most difficult but most important stage of operation is creating a 
sufficiently stable bone stock for the acetabular shell. So, in our case with definite impaction injury of the femoral head, uncemented THA stands justified. Detailed preoperative planning in the the form of 3D reconstruction CT scan to quantify the acetabular defect and MRI scan to assess the damage to the femur head proved vital. Getting good acetabular shell cover was dealt with femoral head autograft reconstruction of the posterior wall.

\section{CONCLUSION}

Acetabular fractures when neglected and mismanaged pose various kinds of technical challenges to the operating surgeon. THA stays the mainstay in management in old age and in young cases with predictably poor outcome to attempted reduction and fixation attempts. Adequate planning and imaging are required in managing a neglected acetabular fracture if an arthroplasty is planned. It becomes an absolute indication in the settings of post traumatic arthritis and femoral head or acetabular impaction providing excellent pain relief and functional improvements. The use of modern implants and alternative bearing surfaces has further improved outcomes.

Funding: No funding sources Conflict of interest: None declared

Ethical approval: Not required

\section{REFERENCES}

1. Bircher M, Lewis A, Halder S. Delays in definitive reconstruction of complex pelvic and acetabular fractures. J Bone Joint Surg Br. 2006;88(9):113740 .
2. Madhu R, Kotnis R, Al-Mousawi A, Barlow N, Deo $\mathrm{S}$, Worlock P, Willett K. Outcome of surgery for reconstruction of fractures of the acetabulum. The time dependent effect of delay. J Bone Joint Surg Br. 2006;88(9):1197-203.

3. Wu ES, Jauregui JJ, Banerjee S, Cherian JJ, Mont MA.Expert Rev Med Devices. 2015;12(3):297-306.

4. Hamlin K, Lazaraviciute G, Koullouros M, Chouari T, Stevenson IM, Hamilton SW. Should Total Hip Arthroplasty be Performed Acutely in the Treatment of Acetabular Fractures in Elderly or Used as a Salvage Procedure Only? Indian J Orthop. 2017;51(4):421-33.

5. Letournel E. Surgical repair of acetabular fractures more than three weeks after injury, apart from total hip replacement. Int Orthop. 1979;2(4):305-13.

6. Sen RK, Veerappa LA. Long-term outcome of conservatively managed displaced acetabular fractures. J Trauma. 2009;67(1):155-9.

7. Veerappa, LA, Tripathy SK, Sen RK. Management of neglected acetabular fractures. Eur J Trauma Emerg Surg. 2015;41:343.

8. Berry DJ, Halasy M. Uncemented acetabular components for arthritis after acetabular fracture. Clin Orthop Relat Res. 2002;405:164-7.

9. Bellabarba C, Berger RA, Bentley CD, Quigley LR, Jacobs JJ, Rosenberg AG, Sheinkop MB, Galante JO. Cementless acetabular reconstruction after acetabular fracture. J Bone Joint Surg Am. 2001;83(6):868-76.

Cite this article as: Avtar R, Khanna V, Kumar K. Neglected posterior acetabular wall fracture managed with uncemented total hip arthroplasty with posterior wall reconstruction by femoral head autograft: a case report. Int J Res Orthop 2018;4:527-31. 